\title{
Ribotype diversity of porcine Pasteurella multocida from Australia
}

\author{
RE BOWLES, JL PAHOFF, BN SMITH and PJ BLACKALL \\ Austral asian Pig Institute, Animal Research Institute, 665 Fairfield Road, Yeerongpilly, Q ueensland 4105 \\ e-mail bowlesr@dpi.qld.gov.au
}

\begin{abstract}
Objective To use the technique of ribotyping to investigate the genetic diversity of Australian isolates of Pasteurella multocida associated with outbreaks of clinical disease in Australian pigs.
\end{abstract}

Design One hundred and seven porcine $P$ multocida isolates were analysed by ribotyping using the restriction enzymes Hpall and Hindll. The genetic population structure of the Australian porcine $P$ multocida isolates was determined through statistical analysis of the joint ribotype patterns, and this was then compared with biochemical and epidemiological data available for the population.

Results A total of 25 combined ribotypes were recognised, which were grouped into five ribotype clusters. Despite the deliberate selection of diverse isolates, the study revealed only a limited degree of genetic diversity. Fourteen of the ribotypes contained multiple isolates, and 12 of these ribotypes were present on more than one farm. Three of the seven biovars analysed in the study showed very limited diversity. All fifteen biovar 2 isolates (subsp multocida) were found in a single cluster (III), while all four biovar 8 isolates, which correspond to $P$ multocida subsp gallicida, were allocated by themselves to a single cluster (IV). All nine of the biovar 12 isolates (lactose-positive subsp multocida) were assigned to a single cluster (I), together with the single biovar 14 isolate, which was the only other lactose-positive isolate in the population (ODC-negative).

Conclusion A limited number of ribotypes of $P$ multocida are associated with Australian pigs. The majority of these ribotypes are widely distributed across multiple farms, and across multiple states. Individual farms can possess multiple ribotypes of $P$ multocida. Some of the unusual biochemical variants of $P$ multocida present in Australian pigs have a very limited genetic diversity. The nature of pig production in Australia, primarily involving continuous flow systems with few closed herds, has possibly contributed to the widespread distribution of a limited number ribotypes.

Aust Vet J 2000;78:630-635

Key Words: Pigs, Pasteurella multocida, ribotype, diversity

\begin{tabular}{ll}
\hline DIG & Digoxigenin \\
ODC & Ornithine decarboxylase \\
PAR & Progressive atrophic rhinitis
\end{tabular}

$\mathrm{P}$ asteurella multocida is a major pathogen of pigs and is involved in a number of different disease conditions. Pneumonic pasteurellosis, one of the most common and widespread diseases of intensively housed pigs ${ }^{1}$ and PAR, an important disease of pigs in N orth America and Europe ${ }^{2}$ are the two major disease conditions associated with $\mathrm{P}$ multocida.

Three subspecies within $P$ multocida ( $P$ multocida subsp multocida, $\mathrm{P}$ multocida subsp septica and $\mathrm{P}$ multocida subsp gallicida) are now recognised. ${ }^{3}$ We have recently completed a pheno- typic characterisation of 150 indole-positive Pasteurella-like organisms isolated from diseased Australian pigs, ${ }^{4}$ using a biotyping scheme we originally developed for avian P multocida. ${ }^{5}$ In this recent study, we found $91 \%$ of the isolates were subsp multocida and the remaining $9 \%$ were subsp gallicida. Six biovars were recognised within the Australian subsp multocida isolates (including two that contain only lactose-positive isolates), and a single biovar was recognised within the Australian subsp gallicida isolates. ${ }^{4}$

Ribotyping appears to be a useful tool for investigating genetic relationships between members of the genus Pasteurella. Petersen et al ${ }^{6}$ recently reported that for the classification of maltose positive isolates of Pasteurella, results from ribotyping (using the $\mathrm{H}$ ind III and $\mathrm{H}$ pall enzymes) correlated closely with data generated from phenotypic and 16S rRN A sequencing studies. Ribotyping has also been used extensively to sub-type both porcine and avian isolates of $P$ multocida. Examples of studies on porcine $P$ multocida include the epidemiological studies of $\mathrm{G}$ ardner et al7 and Zhao and colleagues. 8,9

The information generated from studies of genetic diversity can have significant practical outcomes. A knowledge of the genetic structure of the $\mathrm{P}$ multocida population in Australian pig herds will help to assess the value of ribotyping as an epidemiological tool for porcine P multocida. A knowledge of the genetic diversity present in a collection of strains allows the rational selection of strains to evaluate new diagnostic tests or new vaccines. In the current study, we used ribotyping to examine and quantify the diversity present in a collection of Australian porcine isolates of $\mathrm{P}$ multocida. Isolates in this collection were deliberately selected to be as epidemiologically diverse as possible.

To our knowledge this is the first time that a study has attempted to quantify the genetic diversity present in a porcine $\mathrm{P}$ multocida population, although a similar study has been performed at this laboratory on avian $\mathrm{P}$ multocida. ${ }^{10}$

\section{Materials and methods}

Bacteria

A total of 107 isolates of P multocida were used in this study. All were obtained from Australian pigs and all had been phenotypically characterised. ${ }^{4}$ These field isolates had each been assigned to one of 14 biovars and were found to represent two of the P multocida subspecies (subsp multocida and gallicida), on the basis of ODC activity and the ability to ferment seven carbohydrates : arabinose, dulcitol, maltose, sorbitol, galactose, trehal ose and xylose. $4,5 \mathrm{~T}$ he isolates were deliberately selected to be as diverse as possible. Isolates were obtained from $\mathrm{N}$ ew South Wales (19 isolates from 19 farms), Q ueensland (69 isolates from 39 farms) and Victoria (19 isolates from 19 farms). I solates were generally limited to one per farm unless there was some evidence (such as biovar variation) which indicated that the 
isolates may not have been clonal in nature. Table 1 shows the properties of the isolates used in this study.

Bacterial growth and D N A extraction

Isolates were grown overnight at $37^{\circ} \mathrm{C}$ in Brain $\mathrm{H}$ eart Infusion broth (BBL). Following incubation, samples were plated onto sheep blood agar to check for purity. The bacteria were harvested by centrifugation $\left(15,000 \mathrm{~g}, 20 \mathrm{~min}, 4^{\circ} \mathrm{C}\right)$. The resultant pellet was washed in $5 \mathrm{~mL}$ of SE buffer $(150 \mathrm{mM}$ $\mathrm{NaCl}, 100 \mathrm{mM}$ EDTA, pH 8) and duplicate pellets were retained and were held at $-20^{\circ} \mathrm{C}$ until required for $D N A$ extraction. D N A extraction was performed by a standard methodology as described previously. ${ }^{11}$

\section{Ribotyping}

Total D N A $(5 \mu \mathrm{g})$ was digested with both $\mathrm{H}$ pall and $\mathrm{H}$ ind III (Boehringer $M$ annheim) in separate reactions. The digestions were performed in Tris/acetate buffer ${ }^{12}$ using $6.25 \mathrm{U}$ enzyme per $50 \mathrm{~mL}$ reaction. The digestion was performed at $37^{\circ} \mathrm{C}$ for 3 $\mathrm{h}$ and terminated by heating at $65^{\circ} \mathrm{C}$ for $20 \mathrm{~min}$. D N A fragments were separated by horizontal electrophoresis through a $0.7 \%$ agarose gel at $25 \mathrm{~V}$ for $16 \mathrm{~h}$ in Tris/Borate/EDTA electrophoresis buffer. ${ }^{13}$ DIG-labelled DNA M olecular Weight $M$ arker II (Boehringer $M$ annheim) was used as a marker. The D N A from the gel was transferred to a positively charged nylon membrane (Boehringer $M$ annheim) with the VacuG ene $X L$ Vacuum Blotting System (Pharmacia) according to the manufacturer's instructions.

The probe used in the ribotyping was a PCR-generated $16 \mathrm{~S}$ rRN A gene fragment of the type strain of $P$ multocida subsp multocida (N CTC 10322), prepared and used as described previously for $\mathrm{H}$ aemophilus paragallinarum. ${ }^{14}$ Southern hybridisation and detection were performed using the N ucleic Acid $\mathrm{D}$ etection $\mathrm{K}$ it (Boehringer $\mathrm{M}$ annheim) at $68^{\circ} \mathrm{C}$ for both prehybridisation and hybridisation steps and posthybridisation washes.

\section{Analysis of ribotyping patterns}

Fragment patterns for each restriction enzyme were compared visually and distinct patterns were designated as ribotypes. All bands present in the population were identified and each ribotype assigned a band profile with a value of 1 or 0 corresponding to the presence or absence of each band in the population, respectively. 0 nce the binary band profile for each enzyme was elucidated for each ribotype, combined ribotypes were created by bringing together the binary profiles for each individual restriction enzyme into a single extended profile. $A$ matrix describing the designated band profiles for each ribotype was converted to ASCII file data and imported into the N TSYS computer program (Exeter Publishing, Setauket, NY, USA). A similarity matrix was constructed according to the Dice coefficient with the SIM QUAL function of the N TSYS program. A phenogram of genetic similarity was constructed according to the U PG M A clustering strategy using the SAH N clustering tree and TREE DISPLAY functions of NTSYS. The cophenetic correlation co-efficient was calculated using the M XCOM P function of NTSYS.

\section{Results}

Both restriction enzymes produced well-resolved patterns that contained five to seven bands and allowed easy visual distinctions to be made between the strains. The use of $\mathrm{H}$ indl II gave
13 unique ribotypes among the 107 isolates whereas $\mathrm{H}$ pall gave 12 unique ribotypes. The combination of the two enzyme patterns resulted in 25 unique ribotypes, numbered 1 to 25 . The allocation of the isolates to the ribotypes is shown in Table 1. The details of the ribotype bands and their distribution within the combined ribotypes is outlined in Table 2.

A phenogram of genetic relatedness within the $P$ multocida population, based on the combined ribotyping results, is presented in Figure 1. The cophenetic correlation coefficient for this phenogram was found to have a value of 0.82 , indicating that it was a good reflection of the raw similarity data. Five phenotypically coherent major clusters, numbered I to V, are apparent at a genetic similarity of 0.7 . The properties of these five clusters are shown in Table 3.

Of the 25 ribotypes recognised in this study, 14 contained more than one isolate. W ithin these 14 multi-isolate ribotypes, 12 consisted of isolates from more than one farm. Of these 12 multi-farm ribotypes, three consisted of isolates from farms in just one State, five consisted of isolates from farms in two States and four consisted of isolates from farms in three States. All five ribotype clusters were present on at least two farms, while three clusters (I, II and III) were present on farms in all three States represented in the study.

Of the 14 ribotypes that contained more than one isolate, six consisted of multiple biovars while eight consisted of only one biovar. All 14 multi-isolate ribotypes contained only a single subspecies. While three of the five clusters (I, II and III) contained multiple biovars, clusters IV and V contained only a single biovar, 8 and 3 respectively. Each of the five ribotype clusters contained only one subspecies.

Four unusual biovars were present in the isolates used in this study: biovar 8 (which corresponds to subsp gallicida), biovars 12 and 14 (which correspond to lactose-positive subsp multocida and lactose-positive, ODC-negative subsp multocida) and biovar 13 (which corresponds to OD C-negative subsp multocida).

All four biovar 8 isolates were assigned to a single ribotype cluster (IV), which contained only that biovar. This cluster was markedly distinct from the remainder of the population, and was most closely related to the two isolates that comprised ribotype cluster $\mathrm{V}$.

All nine biovar 12 isolates and the sole biovar 14 isolate were assigned to ribotype cluster I. Seven of the nine biovar 12 isolates were assigned to a single ribotype (ribotype 1). These seven isolates were obtained from seven different farms, with these farms being located in all three States represented in the study.

A total of 16 farms were represented by more than one isolate, and half of these had more than one biovar present. Of the eight farms with more than one biovar all were shown to have isolates that bel onged to different ribotypes. In addition, six of the eight had isolates from different ribotype clusters. Of the eight farms with multiple isolates of a single biovar, three were shown to have isolates that belonged to different ribotypes and one had isolates that belonged to different ribotype clusters.

\section{Discussion}

This study was undertaken to examine the diversity present in Australian isolates of porcine $P$ multocida. For this reason, the collection of isolates examined was deliberately selected to be as diverse as possible. This was done by generally selecting only one isolate from a piggery. In some cases where a prior 
Table 1. Properties of isolates used in this study.

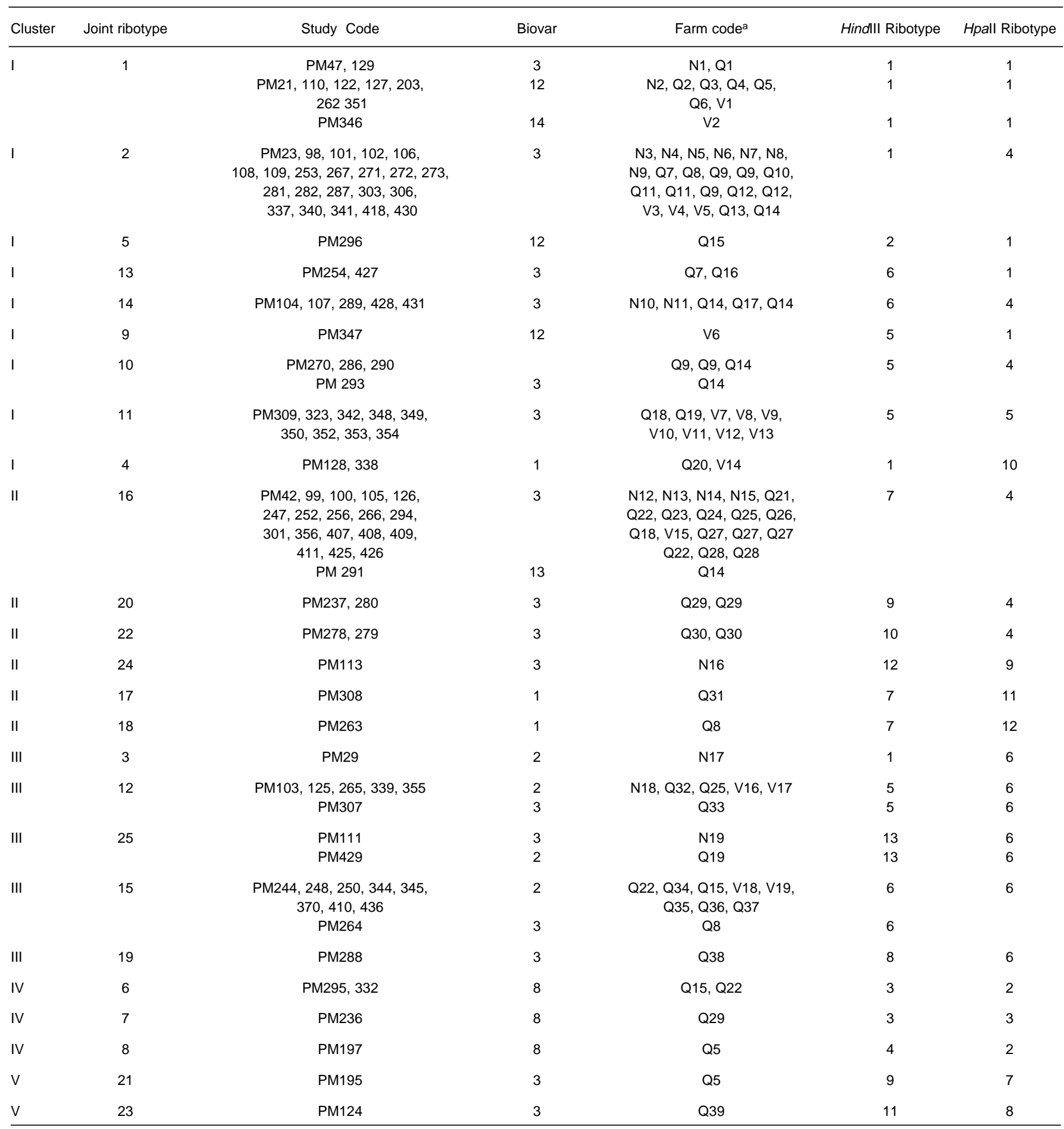

$\mathrm{a}_{\mathrm{N}}=$ New South Wales, $\mathrm{Q}=$ Queensland, $\mathrm{V}=$ Victoria (number indicates individual farms within each state).

biotyping study 4 had indicated diversity existed within isolates of $\mathrm{P}$ multocida from a single piggery, isolates representing some of the different biovars were included. A matching number of piggeries with multiple isolates that did not show biovar variation were also included. H ence, of the 77 farms represented in the study, only 16 farms had multiple isolates. $0 \mathrm{f}$ these 16 farms with multiple isolates, eight had isolates that were known to vary in biovar (a total of 26 isolates) and eight had isolates of the same biovar (a total of 20 isolates).
D espite this strategy of deliberately selecting diverse isolates, the majority of the ribotypes recognised in this study (14 of 25) consisted of multiple isolates. Further, 12 of the 25 ribotypes consisted of isolates from multiple farms. These results suggest that a relatively limited number of types of $P$ multocida are present in the Australian pig herd. In contrast, a recent study with restriction endonuclease analysis of $10 \mathrm{P}$ multocida isolates from pneumonic lung lesions in Australian pigs ${ }^{15}$ showed that there was considerable heterogeneity amongst these isolates. 
Table 2. Band size and distribution among the 25 combined HindIII and HpaII ribotypes of $P$ multocida revealed in this study.

\begin{tabular}{|c|c|c|c|c|c|c|c|c|c|c|c|c|c|c|c|c|c|c|c|c|c|c|c|c|c|c|}
\hline \multirow{2}{*}{$\begin{array}{l}\text { Combined } \\
\text { ribotype } \\
\text { bands }^{\mathrm{a}}\end{array}$} & \multirow{2}{*}{$\begin{array}{c}\text { Molecular } \\
\text { weight }\end{array}$} & \multicolumn{25}{|c|}{ Combined ribotypes $^{b}$} \\
\hline & & 1 & 2 & 3 & 4 & 5 & 6 & 7 & 8 & 9 & 10 & 11 & 12 & 13 & 14 & 15 & 16 & 17 & 18 & 19 & 20 & 21 & 22 & 23 & 24 & 25 \\
\hline $\mathrm{Cl}(\mathrm{Hdl})$ & 12451 & 1 & 1 & 1 & 1 & 1 & 1 & 1 & 1 & 1 & 1 & 1 & 1 & 1 & 1 & 1 & 1 & 1 & 1 & 0 & 1 & 1 & 1 & 1 & 1 & 1 \\
\hline $\mathrm{C} 2(\mathrm{Hd} 2)$ & 9938 & 1 & 1 & 1 & 1 & 1 & 0 & 0 & 0 & 1 & 1 & 1 & 1 & 1 & 1 & 1 & 1 & 1 & 1 & 1 & 1 & 1 & 1 & 0 & 1 & 1 \\
\hline $\mathrm{C} 3(\mathrm{Hpl})$ & 9118 & 0 & 1 & 1 & 1 & 0 & 0 & 0 & 1 & 0 & 1 & 1 & 1 & 0 & 1 & 1 & 1 & 1 & 1 & 1 & 1 & 0 & 1 & 0 & 1 & 1 \\
\hline $\mathrm{C} 4(\mathrm{Hd} 3)$ & 8895 & 0 & 0 & 0 & 0 & 0 & 0 & 0 & 0 & 0 & 0 & 0 & 0 & 0 & 0 & 0 & 0 & 0 & 0 & 0 & 0 & 0 & 0 & 0 & 1 & 0 \\
\hline $\mathrm{C} 6$ (Hp3) & 8046 & 0 & 0 & 0 & 0 & 0 & 0 & 1 & 0 & 0 & 0 & 0 & 0 & 0 & 0 & 0 & 0 & 0 & 0 & 0 & 0 & 0 & 0 & 0 & 0 & 0 \\
\hline $\mathrm{C7}(\mathrm{Hd} 4)$ & 7743 & 0 & 0 & 0 & 0 & 0 & 0 & 0 & 0 & 1 & 1 & 1 & 1 & 0 & 0 & 0 & 0 & 0 & 0 & 1 & 1 & 1 & 0 & 1 & 0 & 1 \\
\hline C8 (HdS) & 7544 & 0 & 0 & 0 & 0 & 0 & 0 & 0 & 0 & 0 & 0 & 0 & 0 & 0 & 0 & 0 & 0 & 0 & 0 & $\mathbf{I}$ & 0 & 0 & 0 & 0 & 0 & 0 \\
\hline $\mathrm{C} 9$ (Hd6) & 7253 & 1 & 1 & 1 & 1 & 1 & 1 & 1 & 1 & 0 & 0 & 0 & 0 & 1 & 1 & 1 & 1 & 1 & 1 & 0 & 1 & 1 & 1 & 1 & 1 & 1 \\
\hline $\mathrm{C} 10(\mathrm{Hp} 4)$ & 7253 & 1 & 1 & 0 & 0 & I & 0 & 0 & 0 & 1 & 1 & 0 & 0 & 1 & 1 & 0 & 1 & 0 & 1 & 0 & 1 & 0 & 1 & 0 & 1 & 0 \\
\hline $\mathrm{C} 11$ (Hd7) & 7063 & 0 & 0 & 0 & 0 & 0 & 1 & 1 & 0 & 0 & 0 & 0 & 0 & 1 & 1 & 1 & 1 & 1 & 1 & 1 & 0 & 0 & 0 & 0 & 0 & 0 \\
\hline $\mathrm{C} 12$ (Hp5) & 6875 & 0 & 0 & 0 & 0 & 0 & 0 & 0 & 0 & 0 & 0 & 0 & 0 & 0 & 0 & 0 & 0 & 0 & 0 & 0 & 0 & 1 & 0 & 0 & 0 & 0 \\
\hline $\mathrm{C} 13$ (Hd8) & 6690 & 1 & 1 & 1 & 1 & 1 & 0 & 0 & 1 & 1 & 1 & 1 & 1 & 0 & 0 & 0 & 0 & 0 & 0 & 0 & 0 & 0 & 0 & 0 & 0 & 0 \\
\hline C16 (HdIO) & 5802 & 0 & 0 & 0 & 0 & 0 & 1 & 1 & 1 & 0 & 0 & 0 & 0 & 0 & 0 & 0 & 1 & 1 & 1 & 0 & 1 & 1 & 1 & 1 & 1 & 0 \\
\hline $\mathrm{C} 17(\mathrm{Hp} 7)$ & 5631 & 0 & 0 & 0 & 0 & 0 & 0 & 0 & 0 & 0 & 0 & 0 & 0 & 0 & 0 & 0 & 0 & 0 & 0 & 0 & 0 & 0 & 0 & 0 & 1 & 0 \\
\hline $\mathrm{C} 18$ (Hp8) & 5378 & 0 & 0 & 1 & 0 & 0 & 0 & 0 & 0 & 0 & 0 & 0 & 1 & 0 & 0 & 1 & $n$ & 1 & 0 & 1 & 0 & 0 & 0 & 0 & 0 & 1 \\
\hline $\mathrm{C} 19$ (Hd11) & 4805 & 0 & 0 & 0 & 0 & 1 & 0 & 0 & 1 & 0 & 0 & 0 & 0 & 0 & 0 & 0 & 0 & 0 & 0 & 0 & 0 & 0 & 1 & 0 & 0 & 0 \\
\hline $\mathrm{C} 20(\mathrm{Hd} / 2)$ & 4487 & 1 & 1 & 1 & 1 & 0 & 1 & 1 & 0 & 1 & 1 & 1 & 1 & 1 & 1 & 1 & 1 & 1 & 1 & 1 & 1 & 1 & 0 & 1 & 1 & 1 \\
\hline C21 (Hp9) & 2987 & 0 & 0 & 1 & 0 & 0 & 1 & 1 & 1 & 0 & 0 & 0 & 1 & 0 & 0 & 1 & 0 & 0 & 0 & 1 & 0 & 1 & 0 & 0 & 0 & 1 \\
\hline $\mathrm{C} 22(\mathrm{Hp} 10)$ & 958 & 0 & 0 & 0 & 0 & 0 & 1 & 1 & 1 & 0 & 0 & 0 & 0 & 0 & 0 & 0 & 0 & 0 & 0 & 0 & 0 & 0 & 0 & 0 & 0 & 0 \\
\hline $\mathrm{C} 23$ (Hp11) & 831 & 1 & 1 & 1 & 1 & 1 & 0 & 0 & 0 & 1 & 1 & 1 & 1 & 1 & 1 & 1 & 1 & 1 & 1 & 1 & 1 & 1 & 1 & 1 & 1 & I \\
\hline $\mathrm{C} 24(\mathrm{Hp} 12)$ & 578 & 0 & 0 & 0 & 1 & 0 & 0 & 0 & 0 & 0 & 0 & 0 & 0 & 0 & 0 & 0 & 0 & 1 & 1 & 0 & 0 & 0 & 0 & 0 & 1 & 0 \\
\hline $\mathrm{C} 25\left(\mathrm{H}_{\mathrm{p}} 13\right)$ & 391 & 1 & 1 & 1 & 1 & 1 & 1 & 1 & 1 & 1 & 1 & 1 & 1 & 1 & 1 & 1 & 1 & 1 & 1 & 1 & 1 & 1 & 1 & 1 & 1 & 1 \\
\hline $\mathrm{C} 26$ (Hp14) & $<100$ & 1 & 1 & 0 & 0 & 1 & 0 & 0 & 0 & 1 & 1 & 1 & 0 & 1 & 1 & 0 & 1 & 0 & 0 & 0 & 1 & 0 & 1 & 0 & 1 & 0 \\
\hline
\end{tabular}

${ }_{\mathrm{b}}^{\mathrm{a}} \mathrm{Bands}$ numbered in order of size, $\mathrm{Hd}=$ present in HindIII ribotype, $\mathrm{Hp}=$ present in $\mathrm{HpaII}$ ribotype.

$\mathbf{b}_{0=\text { Ribotype band absent, } 1 \text { = Ribotype band present. }}^{\text {Bands }}$

H owever the small number of isolates used in this study and the fact that no quantitative estimate of diversity was performed, makes comparisons with the ribotyping data generated from the present study difficult.

While there is no specific knowledge regarding the manage ment practices of all the farms included in the study, the majority of Australian pig farms are a continuous flow type operation and few herds are closed. It is likely that this style of management, combined with the movement of live pigs onto farms, has resulted in the wide distribution of ribotypes across multiple farms.

Three of the seven biovars represented in this study showed limited genetic diversity. The genetic diversity present in biovar 2 was very limited, with all fifteen isolates distributed among four of the five ribotypes present in cluster III. The majority of the biovar 2 isolates were found in only two of the ribotypes, with six placed in ribotype 12 and a further six placed in ribotype 15. All of the biovar 2 isolates originated from different farms, and the farms were located in Q ueensland, N ew South Wales and Victoria. These results suggest that biovar 2 isolates may form a distinct genetic group within P multocida subsp multocida. Interestingly, four biovar 3 isolates comprised the remainder of cluster III, and three of these isolates shared ribotypes with the biovar 2 isolates

The diversity present in biovar 12 , containing lactose positive variants of $P$ multocida subsp multocida, was limited. Seven of the nine isolates were placed in a single ribotype (ribotype 1), with all nine isolates being placed in the same ribotype cluster (cluster I). Of the seven isolates placed in ribotype 1, all seven were from different farms, with the farms being located in $\mathrm{Q}$ ueensland, $\mathrm{N}$ ew South Wales and Victoria. There is no known common connection between all seven piggeries. Interestingly

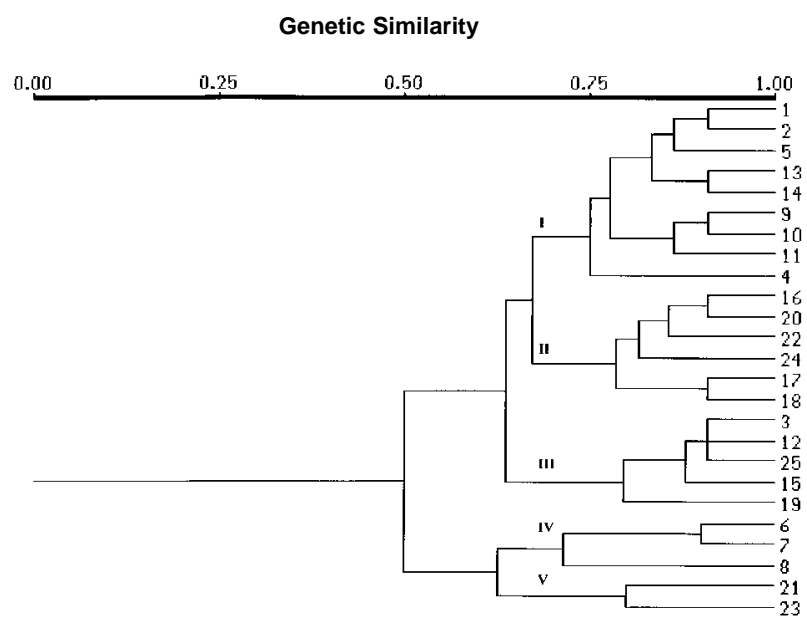

Figure 1. Phenogram of genetic distance amongst 25 ribotypes (numbered 1-25) of 107 porcine isolates of $P$ multocida clustered by the UPGMA strategy. The five major ribotype clusteers (I to V) are marked. Full details are provided in Table 1.

the other lactose positivebiovar, biovar 14, which is also OD Cnegative, was also placed in the same ribotype (ribotype 1) as the majority of the biovar 12 isolates. The limited diversity within the lactose-positive isolates of $\mathrm{P}$ multocida is in contrast to the diversity seen within the isolates previously characterised as maltose-positive P multocida examined by Petersen et al. ${ }^{6}$ These maltose-positive isolates were shown to be very diverse, representing at least three separate species within the genus Pasteurella. ${ }^{6}$

Lactose-positive variants of $P$ multocida subsp multocida have not been widely reported before. Indeed, the only reports of this 
Table 3. Properties of the Pasteurella multocida isolates in the ribotype clusters.

\begin{tabular}{|c|c|c|c|c|c|c|}
\hline Ribotype Cluster & No. of Isolates & Ribotypes & No. of Farms & States & Biovara & Subspecies ${ }^{a}$ \\
\hline I & 56 & $\begin{array}{c}1,2,4,5,9,10,11 \\
13,14\end{array}$ & 45 & QLD, NSW, VIC & $\begin{array}{c}1(3) ; 3(43) \\
12(9) \\
14(1)\end{array}$ & $\begin{array}{c}\text { multocida (46) } \\
\text { lactose }{ }^{+} \text {multocida (9) } \\
\text { lactose }^{+} \text {ODC }^{-} \text {multocida (1) }\end{array}$ \\
\hline ॥ & 26 & $16,17,18,20,22,24$ & 20 & QLD, NSW, VIC & $\begin{array}{c}1(2) ; 3(23) ; \\
13(1)\end{array}$ & $\begin{array}{c}\text { multocida (25) } \\
\text { ODC- multocida (1) }\end{array}$ \\
\hline III & 19 & $3,12,15,19,25$ & 19 & QLD, NSW, VIC & $2(15) ; 3(4)$ & multocida (19) \\
\hline IV & 4 & $6,7,8$ & 4 & QLD & $8(4)$ & gallicida (4) \\
\hline V & 2 & 21,23 & 2 & QLD & $3(2)$ & multocida (2) \\
\hline
\end{tabular}

Number in brackets is the number of isolates of that biovar or subspecies.

variant in pigs have been from Australia and Vietnam, with the latter being a single strain only. 4,16,17 Bisgaard et al ${ }^{18}$ have noted that some isolates of the subspecies multocida and septica obtained from calf lungs were lactose-positive. Given the rare reporting of lactose-positive isolates in pigs, our finding of nine lactose-positive biovar 12 isolates of very limited genetic diversity in Australian pigs is unusual. These isolates may be representative of a genotype that has found a common ecotypic niche in the environment, or alternatively may represent a genotype that has become widespread after originating from a point source.

The second biovar that showed very limited genetic diversity is biovar 8, P multocida subsp gallicida. The only four isolates of this biovar present in the study were found to represent three ribotypes (which possessed only minor pattern variations). These ribotypes were all placed in a single ribotype cluster (IV) that was genetically quite distinct from the majority of the rest of the $\mathrm{P}$ multocida study population (Clusters I to III). There is no known connection between the four Q ueensland piggeries that yielded these four isolates. I solates of subsp galli cida are not common in pigs, with the case report of Cameron et al ${ }^{16}$ and our previous phenotypic study 4 being the only reports of this subspecies in pigs. There have been few previous studies on the genetic diversity of isolates of subsp gallicida. A study of avian isolates of this subspecies found all five Australian isolates belonged to a single ribotype, ${ }^{10}$ which supports the findings of the current study that isolates within subsp gallicida possess limited diversity. In contrast with our finding that the porcine gallicida isolates were quite distinct from the other $P$ multocida isolates by ribotyping, the avian isolates were not markedly different from the other avian isolates by either ribotyping or multi-locus enzyme electrophoresis typing. ${ }^{10} 0$ verall, however, it is apparent that both avian and porcine Australian isolates of P multocida subsp gallicida show limited genetic diversity. O ne of the gallicida isolates included in the current study was isolated as the causative agent of a very severe outbreak of septicaemia that killed over 2000 pigs. ${ }^{16}$ As this study has suggested that all available Australian isolates of $P$ multocida subsp gallicida are genetically closely related, further studies investigating the pathogenic potential for this subspecies in pigs would appear worthwhile.

There were two ODC -negativeisolates included in this study: one of biovar 14 (lactose-positive and ODC-negative) and one of biovar 13 (just ODC-negative). As the biovar 14 isolate was placed in the same ribotype as the majority of the lactose-positive biovar 12 isolates while the biovar 13 isolate was placed in a different ribotype cluster, the biovar 13 and 14 isolates are clearly not closely related. This ability to establish how closely isolates are related is an important advantage of the use of ribotype clustering.

Ribotyping appears to be a useful tool for investigating genetic relationships between members of the genus Pasteurella. Petersen et $a^{6}$ recently reported that, with maltose-positive isolates of Pasteurella, the results from ribotyping (using the $\mathrm{H}$ indl II and $\mathrm{H}$ pall enzymes) correlated closely with data generated from phenotypic and 16S rRN A gene sequencing studies. In our study, we have found some disagreement between classification into biovars and ribotypes within $\mathrm{P}$ multocida subsp multocida. Unlike our previous study on avian isolates, ${ }^{10}$ we found that with the porcine isolates, almost half of the multiisolate ribotypes ( 6 of 14) contained multiple biovars. H owever our current study has also found that of the eight farms that had multiple isolates of different biovars, ribotyping confirmed that multiple ribotypes were present on all eight farms. For the eight farms with multiple isolates of the same biovar, ribotyping identified multiple ribotypes in three. Based upon the methods described in this study, ribotyping is a better tool than biotyping for the purpose of establishing if different "types" of $P$ multocida are present on a farm. 0 ur results indicate that, in the absence of a capacity to perform ribotyping, biotyping may give some idea of the diversity of $P$ multocida present in a pig herd, al though the use of biotyping is likely to give an under-estimate of diversity.

In conclusion, this study has provided new knowledge on the diversity of Australian porcine isolates of $P$ multocida. We were able to recognise only a relatively limited number of ribotypes, despite deliberately selecting a diverse collection of isolates. M ost of the ribotypes consisted of isolates from multiple farms. These discoveries suggest that a limited number of ribotypes have been widely distributed across pig farms in Eastern Australia, and this may have been due to management practices that have permitted the movement of live pigs between herds.

We have established that some of the unusual biochemical variants of $\mathrm{P}$ multocida present in Australian pigs have a very limited genetic diversity.

\section{Acknowledgments}

The assistance of M S Michelle Adamson (Victorian Department of Agriculture), M r Paul Duffy (Yeerongpilly Veterinary Laboratory), M s Carol Stephens (Toowoomba Veterinary Laboratory) and Dr M ark W hite (Allied Feeds) in providing some of the isolates used in this study is gratefully acknowledged. The assistance of M s J eanette M iflin in the synthesis and provision of the ribotyping probe is gratefully 
acknowledged. This work was funded, in part, by the Pig Research and D evelopment Corporation.

\section{References}

1. Pijoan C. Pneumonic pasteurellosis. In: Leman AD, Straw BE, Mengeling WL, D'Alliare S, Taylor DJ, editors. Diseases of swine. lowa State University Press, Ames, 1992;552-559.

2. De Jong MF. (Progressive) atrophic rhinitis. In: Leman AD, Straw BE, Mengeling WL, D'Alliare S, Taylor DJ, editors. Diseases of swine. lowa State University Press, Ames, 1992;414-435.

3. Mutters R, Ihm P, Pohl S et al. Reclassification of the genus Pasteurella Trevisan 1887 on the basis of deoxyribonucliec acid homology, with proposals for the new species Pasteurella dagmatis, Pasteurella canis, Pasteurella stomatis, Pasteurella anatis, and Pasteurella langaa. Int J Syst Bacterio 1985;35:309-322.

4. Blackall PJ, Pahoff JL, Bowles R. Phenotypic characterisation of Pasteurella multocida isolates from Australian pigs. Vet Microbiol 1997;57:355-360.

5. Fegan N, Blackall PJ, Pahoff JL. Phenotypic characterisation of Pasteurella multocida isolates from Australian poultry. Vet Microbiol 1995;47:281-286.

6. Petersen KD, Christensen JP, Bisgaard M. Phenotypic and genotypic diversity of organisms previously classified as maltose positive Pasteurella multocida. Zbl Bakt 1998;288:1-12.

7. Gardner IJ, Kasten R, Eamens GJ et al. Molecular fingerprinting of Pasteurella multocida associated with progressive atrophic rhinitis in swine herds. J Vet Diagn Invest 1994;6:442-447.

8. Zhao G, Pijoan C, Murtaugh MP, Molitor TW. Use of restriction endonuclease analysis and ribotyping to study epidemiology of Pasteurella multocida in closed swine herds. Infect Immun 1992;60:1401-1405.
9. Zhao G, Pijoan C, Murtaugh MP. Epidemiology of Pasteurella multocida in a farrow-to-finish swine herd. Can J Vet Res 1992;57:136-138.

10. Blackall PJ, Fegan N, Chew GTI, Hampson DJ. Population structure and diversity of avian isolates of Pasteurella multocida from Australia. Microbiology 1998;144:279-289.

11. Blackall PJ, Pahoff JL, Marks D et al. Characterisation of Pasteurella multocida isolates from fowl cholera outbreaks on seven turkey farms. Aust Vet $J$ 1995;72:135-138.

12. O'Farrell PH, Kutter E, Nakanishi M. A restriction map of the bacteriophage T4 genome. Mol Gen Genet 1980;179:421-435.

13. Sambrook J, Fritsch EF, Maniatis T. Molecular cloning: a laboratory manual. Cold Spring Harbour Laboratory Press, Cold Spring Harbour, 1989:2000.

14. Miflin JK, Chen X, Blackall PJ. Molecular characterisation of isolates of Haemophilus paragallinarum from China by ribotyping. Avian Pathol 1997;27:119-127.

15. Djordjevic SP, Eamens $\mathrm{GJ}, \mathrm{Ha} \mathrm{H}$ et al. Demonstration that Australian Pasteurella multocida isolates from sporadic outbreaks of porcine pneumonia are non-toxigenic (tox $\left.A_{-}\right)$and display heterogenous DNA restriction endonuclease profiles compared with toxigenic isolates from herds with progressive atrophic rhinitis. J Med Microbiol 1998;47:679-688.

16. Cameron RDA, O'Boyle D, Frost AJ, Fegan N. An outbreak of haemorrhagic septicaemia associated with Pasteurella multocida subsp gallicida in a large pig herd. Aust Vet J 1996;73:27-29.

17. Townsend KM, O'Boyle D, Phan TT et al. Acute septicaemic pasteurellosis in Vietnamese pigs. Vet Microbiol 1998;63:205-215.

18. Bisgaard M, Houghton SB, Mutters R, Stenzel A. Reclassification of German, British and Dutch isolates of so-called Pasteurella multocida obtained from pneumonic calf lungs. Vet Microbiol 1991;26:115-124

(Accepted for publication $22 \mathrm{M}$ arch 2000)

\section{Reducing Salmonella in pigs}

In many developed countries, salmonellosis has become one of the most important meat-transmitted zoonotic diseases. In some studies, over $10 \%$ of Salmonella outbreaks attributed to meat have been associated with pork. Infection may be introduced onto farms by contaminated feed, rodents, fomites and carrier pigs. Efforts to identify and eliminate the carrier pig population are hindered by, amongst other things, the lack of reliable practical means to control the carriage of Salmonella by subclinically infected pigs and limit spread to other pigs in contact with them. At the abattoir, the carrier pig is the initial source of contamination of the environment, other animals and, potentially, meat products. Whereas attention to good hygienic practice at the abattoir is important in avoiding or limiting contamination, removal of infection from herds has the potential to provide a longer term solution.

In an experiment, pigs were challenged with a virulent field isolate of Salmonella Typhimurium after receiving one of a range of treatments. Effect of treatment was assessed by comparing the number of isolations of the organism from various tissues and faeces at 14 days. Salmonella excretion was not reduced by acidification of water, use of specific immunoglobulins or vaccination with an endotoxin vaccine. Number of Salmonella in colonised mesenteric lymph nodes was reduced with the use of bambermycins and a live attenuated vaccine and shedding in faeces was reduced after supplementation with fructo-oligosaccharides in drinking water. Whereas the use of probiotics and prebiotics caused changes in faecal flora this was not associated with a reduction in shedding of Salmonella.

Letellier A, Messier S, Lessard L, Quessy S. Assessment of various treatments to reduce carriage of Salmonella in swine. Can J Vet Res 2000;64:27-31. 